\author{
Sabine Schmidt \\ Patrick Chevallier \\ Marc Chalaron \\ Bertrand Bessoud \\ Francis R. Verdun \\ Philippe Frascarolo \\ Pierre Schnyder \\ Alban Denys
}

\section{Multidetector CT enteroclysis: comparison of the reading performance for axial and coronal views}

Received: 19 August 2004

Revised: 1 October 2004

Accepted: 7 October 2004

Published online: 24 November 2004

(C) Springer-Verlag 2004

S. Schmidt $(\bullet) \cdot$ M. Chalaron

P. Schnyder · A. Denys

Service de radiodiagnostic et radiologie interventionnelle,

Centre Hospitalier Universitaire

Vaudois-CHUV,

Rue du Bugnon, 1011 Lausanne,

Switzerland

e-mail: sabine.schmidt@chuv.hospvd.ch

Tel.: +41-21-3144444

Fax: +41-21-3144443

P. Chevallier

Department of Diagnostic Radiology,

CHU,

Nice, France

B. Bessoud

Department of General Radiology,

BROCA

Le Kremlin-Bicêtre, France

F. R. Verdun

University Institute of Applied Radiophysics,

Lausanne, Switzerland

P. Frascarolo

Department of Anesthesiology,

Lausanne, Switzerland

\begin{abstract}
The purpose of this study was to compare the diagnostic performance of axial and coronal views in multidetector CT enteroclysis (MDCTE). We retrospectively evaluated 48 patients with pathological correlation investigated by MDCTE for small bowel disorders. After nasojejunal administration of 21 of $5 \%$ methylcellulose axial arterial and venous acquisition of MDCTE was followed by coronal reconstructions using equal slice thicknesses of $2.5 \mathrm{~mm}$ with $2 \mathrm{~mm}$ increments. Spatial resolution of both planes was evaluated by phantom. Three radiologists independently read axial and coronal images concerning 12 pathological features. The interobserver agreement and time of reading was calculated. Sensitivity and specificity resulted from comparison with histopathology $(n=39)$ or follow-up $(n=9)$. Phantom study revealed higher spatial resolution for axial than coronal views, whatever reconstruction interval was used. However, spatial frequency always remained
\end{abstract}

high. Most pathological signs, such as bowel wall thickening (BWT), bowel wall enhancement (BWE) and intraperitoneal fluid (IPF), showed better interobserver agreement on axial than coronal views (BWT: 0.61 vs. 0.44; BWE: 0.56 vs. 0.5; IPF:0.53 vs. 0.43 ). The Wilcoxon signed-rank test revealed significantly higher sensitivity for axial than coronal views $(P=0.0453)$; the time of reading was significantly shorter for the latter $(P=0.0146)$. The diagnostic value of axial slices is superior to coronal reconstructions despite the reduced data volume and display of the physiological course of bowel loops on the coronal plane.

Keywords Computed tomography multi-detector row - Intestines · Enteroclysis

\section{Introduction}

The technique of enteroclysis remains the gold standard for the radiological investigation of small bowel disorders [1-4], since only volume challenge results in homogeneous luminal distension, which is mandatory for the detection of intestinal diseases. The additional inherent advantage of cross-sectional imaging modalities such as $\mathrm{CT}$ enteroclysis and MR enteroclysis is the simultaneous depiction of intraluminal, mural and extraintestinal pathologies [2-6]. Spiral CT enteroclysis, which has evolved to an established modality for the investigation of various small bowel disorders [2, 3, 7-12], is now gradually being replaced by multidetector CT enteroclysis (MDCTE) [13-15].

Development of multidetector row technology in 1998 [16], based on the helical CT that was introduced in 1989 [17], now allows data acquisition over the entire abdomen 
in thin slices within one single breath-hold. This multi-slice acquisition not only increases spatial resolution, but also reduces motion artifacts. Therefore, even the most fragile patients can be investigated. Multiplanar reconstructions are secondarily realized within minutes without an increase of time. The coronal plane in particular is better suited to display physiological bowel anatomy, especially the ileocecal valve. It corresponds to the view of the surgeon at laparotomy and is of the same orientation as conventional examinations; therefore, it is well received by the referring clinician. Moreover, the entire abdominal cavity can be displayed in a lower number of slices than in the axial plane, potentially reducing the reading time of the radiologist. Axial slices often demonstrate bowel loops in their short axis, so it is often laborious to follow their course in order to determine the exact length of any mural pathology, especially in segmentary stenoses. The aim of this retrospective study is to compare the diagnostic performance of axial slices in MDCTE with coronal reconstructions.

\section{Material and methods}

Spatial resolution of axial slices and coronal reconstructions

In order to compare the spatial resolution of axial and coronal slices, we performed an experimental study using a quality assurance phantom supplied by the manufacturer of the CT unit (QA phantom GE Medical Systems, Milwaukee, WI). This test object contains a polymethylmetacrylate (PMMA or Plexiglas) bloc with a resolution test pattern including the following spatial frequencies: $0.31 \mathrm{~mm}^{-1}, 0.38 \mathrm{~mm}^{-1}, 0.50 \mathrm{~mm}^{-1}, 0.63 \mathrm{~mm}^{-1}$ and $0.83 \mathrm{~mm}^{-1}$. From the fact that these modulations make an angle of $45^{\circ}$ with the $x$ and $y$ directions (Fig. 1a), their frequencies should be multiplied by $\cos \left(45^{\circ}\right.$; i.e., 0.707$)$ when dealing with their sampling in the $x$ direction and $y$ direction. This PMMA bloc is immersed in water in a cylindrical test object of $21 \mathrm{~cm}$. To be representative of abdominal acquisitions, transverse slices of this test pattern were first acquired using a pitch of 1.5 and a field of view of $36 \mathrm{~cm}$. Matrix size always remained $512 \times 512$. To assess the spatial resolution of the coronal reformatted images, the test object was rotated by $90^{\circ}$. A standard acquisition was then performed using a reconstructed slice thickness of $2.5 \mathrm{~mm}$ with an increment of $2.0 \mathrm{~mm}$. From this set of data, coronal views, $2-\mathrm{mm}$ thick, were reconstructed by means of the MPR algorithm available on the CT unit. This procedure is strictly equivalent to the one applied on the clinical images.

Based on the same acquisition parameters, a second coronal reconstruction was performed using a slice increment of $1.2 \mathrm{~mm}$. Spatial frequency of each of the two differently reconstructed coronal views was then compared with the one of axial slices.

\section{Patients}

Among 180 patients investigated by MDCTE within 31 months in our department, we retrospectively evaluated 48 patients (27 women, $21 \mathrm{men}$, mean age $=48$ years) with pathological correlation. Clinical indications were the following: 30 patients $(63 \%)$ presented with known or suspected inflammatory bowel disease, $10(21 \%)$ with possible small bowel tumors or metastases, 5 $(10 \%)$ with suspected postoperative complications, such as strictures of leakages, $2(4 \%)$ with digestive vascular pathologies and 1 (2\%) with diffuse abdominal complaints.

\section{Technique}

Patients had fasted for $8 \mathrm{~h}$ prior to MDCTE without other digestive preparation. After nasal intubation of the proximal jejunum under fluoroscopy, 21 of 5\% methylcellulose was introduced to the small bowel by a power-injector (Guerbet $\mathrm{GmbH}$, Sulzbach/Taunus, Germany) at a flow rate of $200 \mathrm{ml} / \mathrm{min}$ immediately before MDCTE. All patients received $20 \mathrm{mg}$ of scopolaminbutylbromid (Buscopan, Boehringer Ingelheim, Basel, Switzerland) i.v. or, if contraindicated, $1 \mathrm{mg}$ of glucagon (GlucaGen, Novo Nordisk, Bagsvaerd, Denmark) to reduce peristalsis. After intravenous administration of the contrast medium iopentol (Imagopaque, Nycomed Imaging AS, Oslo, Norway) at a flow rate of $2.5 \mathrm{ml} / \mathrm{s}$ (volume in $\mathrm{ml}=$ body weight $+30 \mathrm{ml}$ ). Multidetector spiral-CT acquisition (four-detector-array, Light Speed QX/i, GE Medical Systems, Milwaukee, WI) was performed within $3 \mathrm{~min}$. We obtained slices in the arterial (at $30 \mathrm{~s}$ ) and venous phase (at $60 \mathrm{~s}$ ) from the diaphragm to pubic symphysis (200-240 mA, voltage $120 \mathrm{kV}$, table speed: $15 \mathrm{~mm} /$ rotation, pitch 1.5 , collimation $4 \times 2.5 \mathrm{~mm}$, axial and coronal slice reconstruction: $2.5 \mathrm{~mm} / 2 \mathrm{~mm}$ ). Advantage Windows 4.0 GE work station served for the postprocessing procedures (MPR).
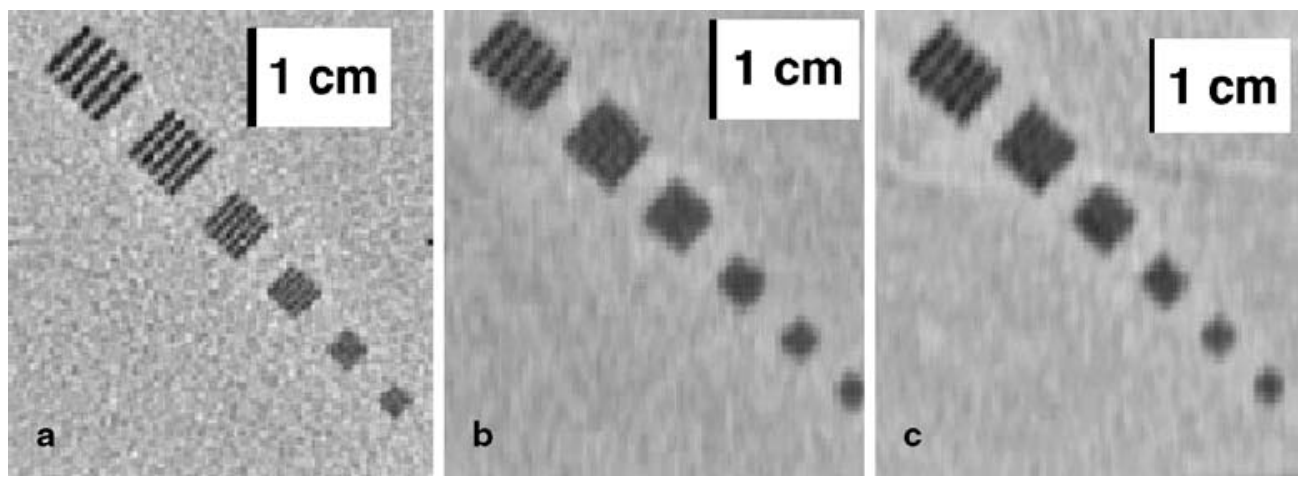

Fig. 1 a Transverse slice of the resolution test pattern of the test object shows a spatial resolution of nearly $0.63 \mathrm{~mm}^{-1}$ (i.e., modulations detectable up to the fourth line pairs group), their acquisition parameters exactly corresponding to those of axial images of MDCTE $($ pitch $=1.5 /$ slice thickness $=2.5 \mathrm{~mm} /$ slice increment $=$ $2 \mathrm{~mm}$ ) b Coronal view of the test object reformatted from axial slices seen in a with an increment of $2 \mathrm{~mm}$ demonstrates that only one group of line pairs can be resolved $\left(0.31 \mathrm{~mm}^{-1}\right)$. c Coronal view of the test object using the same acquisition conditions as in a, but a slice increment of $1.2 \mathrm{~mm}$, leading to minimal change of spatial resolution since still modulations up to $0.31 \mathrm{~mm}^{-1}$ can be detected only 
Proof of disease

The gold standard was defined as the number and anatomical extension of pathological findings seen in consensus on axial slices by the two investigators (AD, SS), who were aware of all the data, always in correlation with patient outcome. Proof of disease was obtained by histopathological findings resulting from subsequent surgery or retrograde endoscopy of the terminal ileum. Segment-by-segment correlation was made between imaging studies and histopathological proof. If there was no operation or simultaneous retrograde endoscopy performed, long-term clinical follow-up was taken into consideration.

For $39(81.3 \%)$ patients, pathological proof of disease was available. The patients' clinical outcomes had to be correlated with radiological features in the remaining nine. Median follow-up was 15 months for the latter.

Image analysis

Three radiologists (BB, MC, $\mathrm{PC}$ ) with extensive experience in abdominal imaging resulting from working in a specialized university hospital independently evaluated the diagnostic findings of axial slices and coronal reconstructions separately in a blinded fashion in search of 12 pathological signs: bowel wall thickening (BWT), bowel stenosis (BST), pathological bowel wall enhancement (BWE) after intravenous injection of contrast medium, intra-/extraluminal mass (IEM), adjacent mesenteric fat infiltration (MFI), lymphadenopathy (ADP), intraperitoneal fluid (IPF), fistula (FIS), abscess (ABS), parietal vascular malformation (PVM), intestinal perforation (INP) and invagination (INV). Radiological features had to be attributed either to one of six bowel segments (jejunum, ileum, cecum/ascending colon, transverse colon, descendent colon and sigmoid colon/rectum) or situated in one of six anatomical abdominal areas (left or right hypochondriac, left or right epigastric region and left or right iliac fossa).

We used a four-point scale to determine observer confidence: The diagnosis was certain $(100 \%)$, probable $(60 \%)$, possible $(30 \%)$ or excluded $(0 \%)$, receiving grades from one (certain) to four (excluded); the first two levels were considered as a positive, the last two levels as a negative result.

Image analysis was essentially filmless, done by cine-display on the work-station. Each radiologist independently and blindly reviewed axial slices in one session followed by reading of coronal reconstructions at a distance of at least 6 weeks in order not eventually to remember any cases, which had also been randomly mixed. Individual time of reading was taken into account for each technique and each patient.

Pathological features were exactly defined as follows: the bowel wall was considered as thickened if there was a parietal diameter of more than $3 \mathrm{~mm}$. Bowel stenosis was present if the luminal narrowing was preceded by prestenotic dilatation. Pathological bowel wall enhancement and mesenteric fat infiltration were defined by their different density or attenuation compared to the surrounding equivalent structures. Mesenteric lymphadenopathy was defined as a lymph node whose smallest diameter was larger than $5 \mathrm{~mm}$. Any intraperitoneal fluid was considered as a pathological feature.

\section{Statistical analysis}

Interobserver agreement between the three radiologists was calculated using kappa statistics concerning each of the 12 pathological features detected on MDCTE. Strength of agreement was characterized according to the proposed ratings by Fleiss [18]: kappa $<0.4=$ poor, $0.40-0.75=$ good and $>0.75=$ excellent agreement. By comparing the result of each of the three radiologists with histopathological results or patients' clinical outcome, we calculated the mean sensitivity and mean specificity for each of the 12 pathological features. THe Wilcoxon signed rank test and paired Student's $t$ test were used to show statistical significance.

\section{Results}

Phantom study: results

In the standard acquisition conditions $(\mathrm{FOV}=36 \mathrm{~cm} / \mathrm{pitch}$ $=1.5 /$ slice thickness $=2.5 \mathrm{~mm} /$ slice increment $=2 \mathrm{~mm}$ ) of the transverse slices of the test object, the pixel size in plane resolution is equal to $0.7 \mathrm{~mm}$, leading to an image sampling frequency of $1.4 \mathrm{~mm}^{-1}$. In such conditions the Nyquist frequency (highest spatial frequency theoretically detectable) is equal to $1.4 / 2=0.7 \mathrm{~mm}^{-1}$. Figure $1 \mathrm{a}$ shows that modulations can be detected significantly under this value, i.e., modulations detectable up to $0.63 \mathrm{~mm}^{-1}$, which corresponds in fact to a frequency of $0.45 \mathrm{~mm}^{-1}$ in the $x$ or $y$ direction.

Using a slice increment of $2 \mathrm{~mm}(\mathrm{FOV}=36 \mathrm{~cm})$, the sampling frequency of the reformatted coronal slices is equal to $0.5 \mathrm{~mm}^{-1}$, leading to a Nyquist frequency of $0.25 \mathrm{~mm}^{-1}$. As shown in Fig. 1b, the spatial frequency of the reformatted images is much lower than the one shown on axial slices produced with a sampling of $1.4 \mathrm{~mm}^{-1}$ (Fig. 1a). The limiting spatial resolution of Fig. $1 \mathrm{~b}$ is now $0.31 \mathrm{~mm}^{-1}$ (only one group can be resolved), which corresponds to a frequency of $0.22 \mathrm{~mm}^{-1}$ in the $x$ or $y$ direction, a value compatible with a Nyquist frequency of $0.25 \mathrm{~mm}^{-1}$.

Secondly, without change of any other parameters, we used a slice increment of $1.2 \mathrm{~mm}$, which thus increased the sampling frequency to $0.83 \mathrm{~mm}^{-1}$, leading to a Nyquist frequency of $0.415 \mathrm{~mm}^{-1}$ (Fig. 1c). However, in spite of almost doubling the sampling frequency, the spatial resolution did not improve very much since it is still only possible to resolve modulations up to $0.31 \mathrm{~mm}^{-1}$, as shown in Fig. 1c. Therefore, we applied the reconstruction algorithm demonstrated in Fig. 1b on our coronal reformatted images.

\section{MDCTE: results}

Time spent on reading by each of the three radiologists is displayed in Table 1. The mean time for axial slices was $7.6 \mathrm{~min}$, which was statistically significantly longer than for coronal reconstructions with $6.6 \mathrm{~min}(P=0.0146)$. In general, the number of axial slices (280-300) was twice

Table 1 Time of reading per patient spent by each observer on axial slices and coronal reconstructions

Time of reading

Radiologist Axial (280-300 slices) Coronal (130-150 slices)

\begin{tabular}{lll}
\hline 1 & $8.4 \mathrm{~min}$ & $6.7 \mathrm{~min}$ \\
2 & $6.7 \mathrm{~min}$ & $6.6 \mathrm{~min}$ \\
3 & $7.5 \mathrm{~min}$ & $6.6 \mathrm{~min}$ \\
Mean & $7.6 \mathrm{~min}$ & $6.6 \mathrm{~min}$ \\
\hline
\end{tabular}


Table 2 Interobserver agreement, sensitivity and specificity of the six most common pathological features resulting from independent, random and blinded evaluation of axial slices and coronal reconstructions by the three radiologists: BWT bowel wall thickening, BST bowel stenosis, BWE pathological bowel wall enhancement, MFI adjacent mesenteric fat infiltration, ADP lymphadenopathy, IPF intraperitoneal fluid

\begin{tabular}{|c|c|c|c|c|c|c|}
\hline $\begin{array}{l}\text { Pathological feature } \\
\text { Technique }\end{array}$ & \multicolumn{2}{|c|}{ Interobserver agreement (kappa) } & \multicolumn{2}{|c|}{ Sensitivity (\%) } & \multicolumn{2}{|c|}{ Specificity (\%) } \\
\hline $\mathrm{BWT}(n=49)$ & 0.61 & 0.44 & 81.6 & 73.3 & 81.7 & 85 \\
\hline BWE $(n=36)$ & 0.56 & 0.5 & 75.9 & 73.7 & 88.9 & 90.6 \\
\hline MFI $(n=37)$ & 0.57 & 0.66 & 77.5 & 65.6 & 97.9 & 95.1 \\
\hline $\operatorname{ADP}(n=36)$ & 0.69 & 0.65 & 85.2 & 79.4 & 96 & 93.5 \\
\hline
\end{tabular}

a Numbers in parentheses are standard deviation.
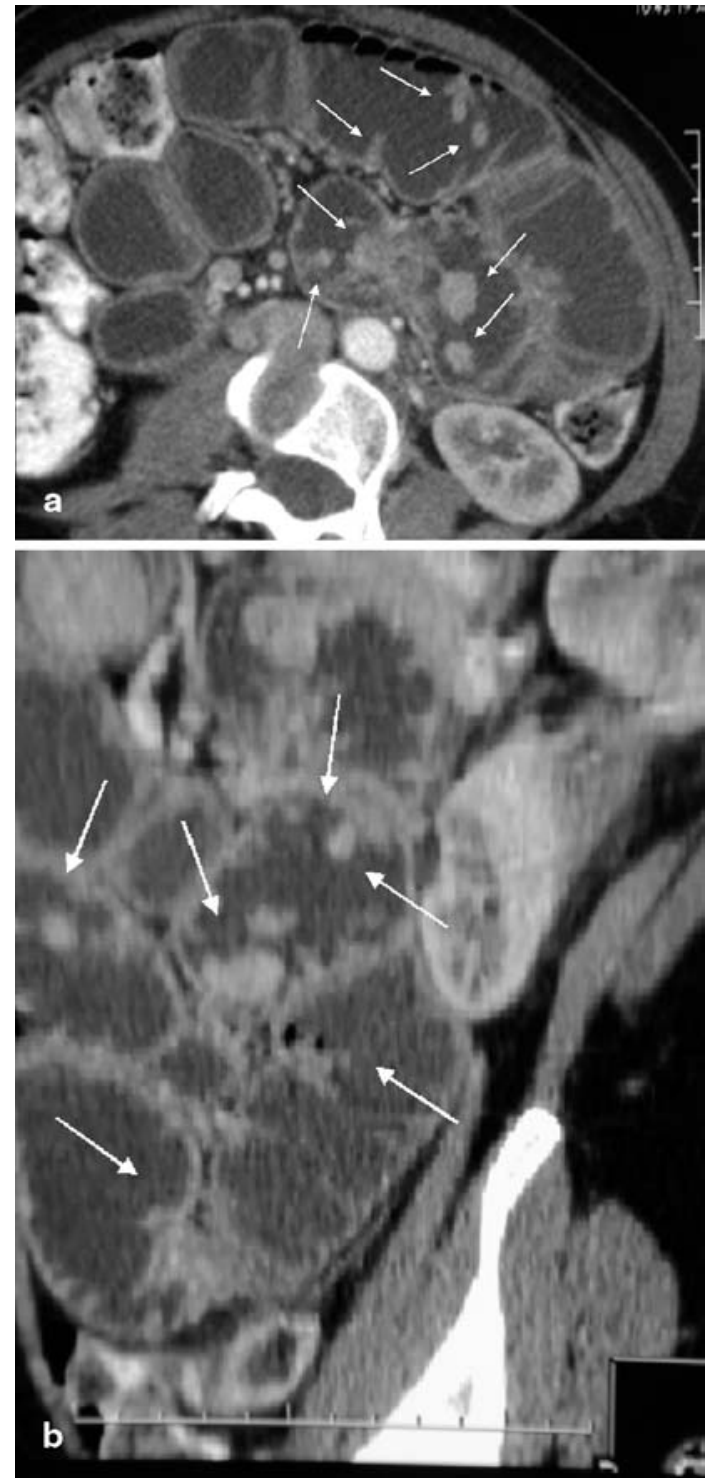

Fig. 2 a,b MDCTE in a 40-year-old woman with Peutz-Jeghers disease: multiple pedunculated jejunal and ileal hamartomas (arrows) are equally detected on $\mathbf{a}$ axial and $\mathbf{b}$ coronal views
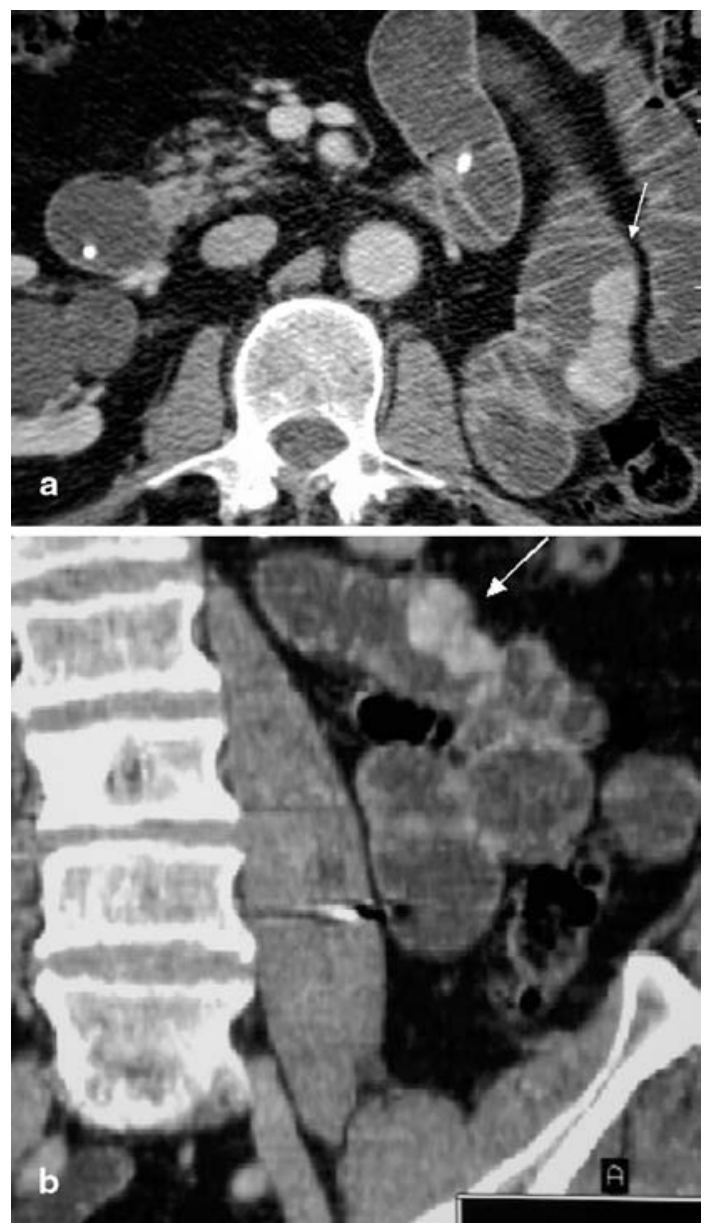

Fig. 3 a,b MDCTE in a 65-year-old man with known renal cell carcinoma, now developing hypervascular metastasis within the proximal jejunum (arrow): optimal luminal distension with neutral contrast medium and thin-slice acquisition allows for clear visualization on a axial slice as well as on $\mathbf{b}$ coronal reconstruction 

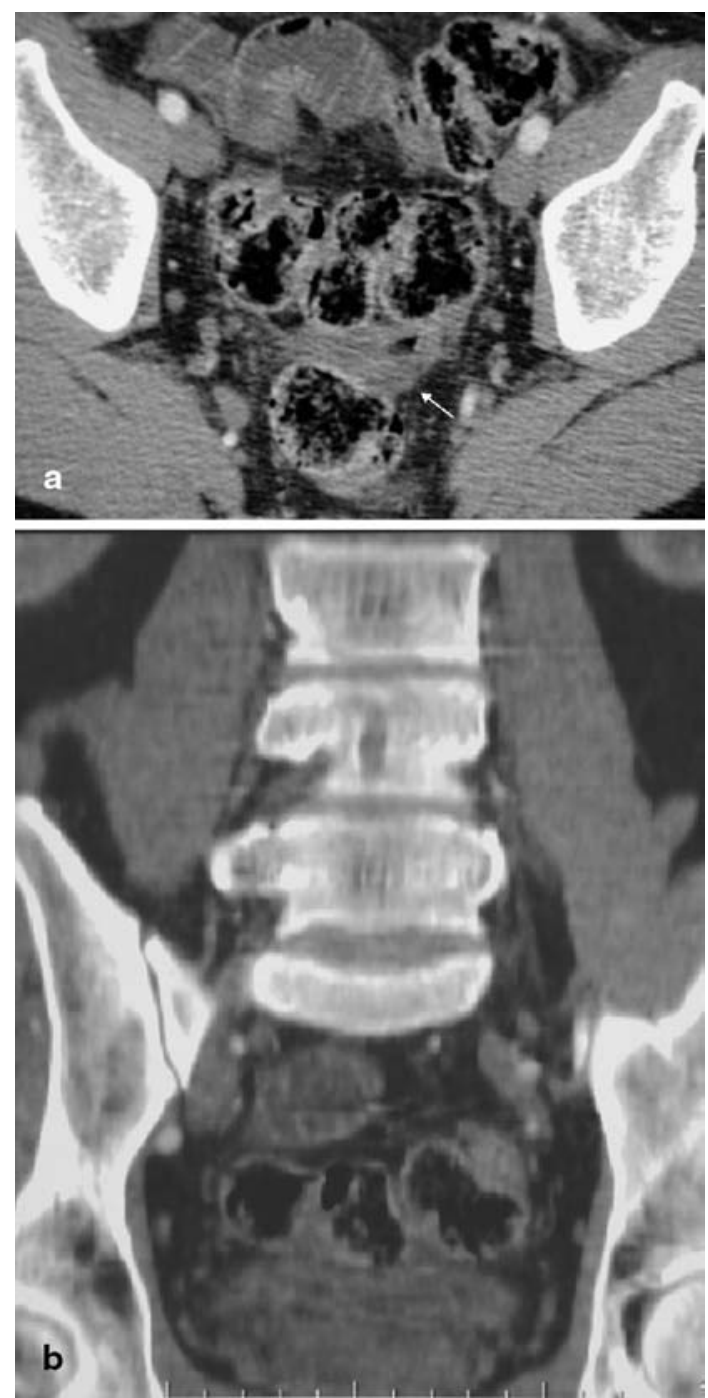

Fig. 4 a,b MDCTE in a 70-year-old man with acute inflammatory bowel disease: small amounts of free pelvic fluid (arrow) seen on a axial views are hardly detected on $\mathbf{b}$ coronal reconstructions

as important compared to those of coronal reconstructions $(130-150)$.

Overall incidence of the 12 pathological signs detected in consensus on axial images by the two observers aware of the pathological and clinical context (see Table 2) was the following: 49 areas of bowel wall thickening (BWT), 24 bowel wall stenoses (BWS) and 36 segments of pathological bowel wall enhancement (BWE). In all these three pathological signs, the ileum was the predominant localization, accounting for $53 \%(n=26)$ in BWT, 68\% (n=17) in BWS and 61\% ( $n=22)$ in BWE. Five intraluminal masses, i.e., primary tumors or metastases (Fig. 2, 3), and four enteral fistulas could be seen equally on both techniques.

One of five abscesses detected on axial slices could not be detected on coronal reconstructions becauses of
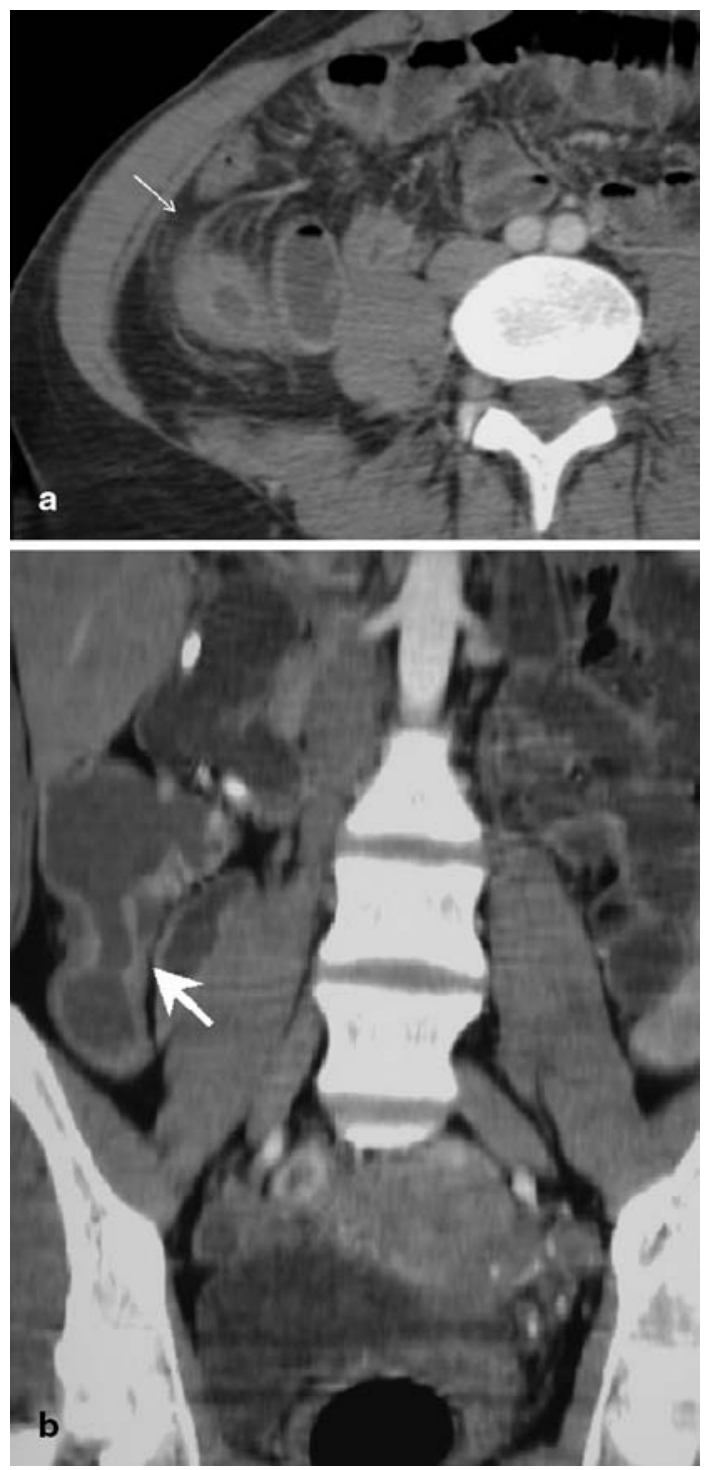

Fig. 5 a,b MDCTE in a 30-year-old woman with intermittent Crohn's disease after ileocaecal resection in the past: signs of acute inflammation, that is, hyperemia of mesenteric vessels and extension of adjacent mesenteric edematous infiltration (thin arrow), are better seen on a axial slices, while the exact length of intestinal stenosis (thick arrow) is better appreciated on b coronal reconstructions

its small size, the superficial location just beneath the abdominal wall and its extension mainly into the transverse plane, which was later confirmed by surgery. Axial slices revealed 36 areas of enlarged lymph nodes and 37 regions of mesenteric fat infiltration; among the latter, six were located in the pelvis without clear visualization on coronal reconstructions. Finally, there were 22 areas of intraperitoneal fluid hardly seen on coronal slices in eight patients probably because of the small amount of pelvic ascites (Fig. 4). 

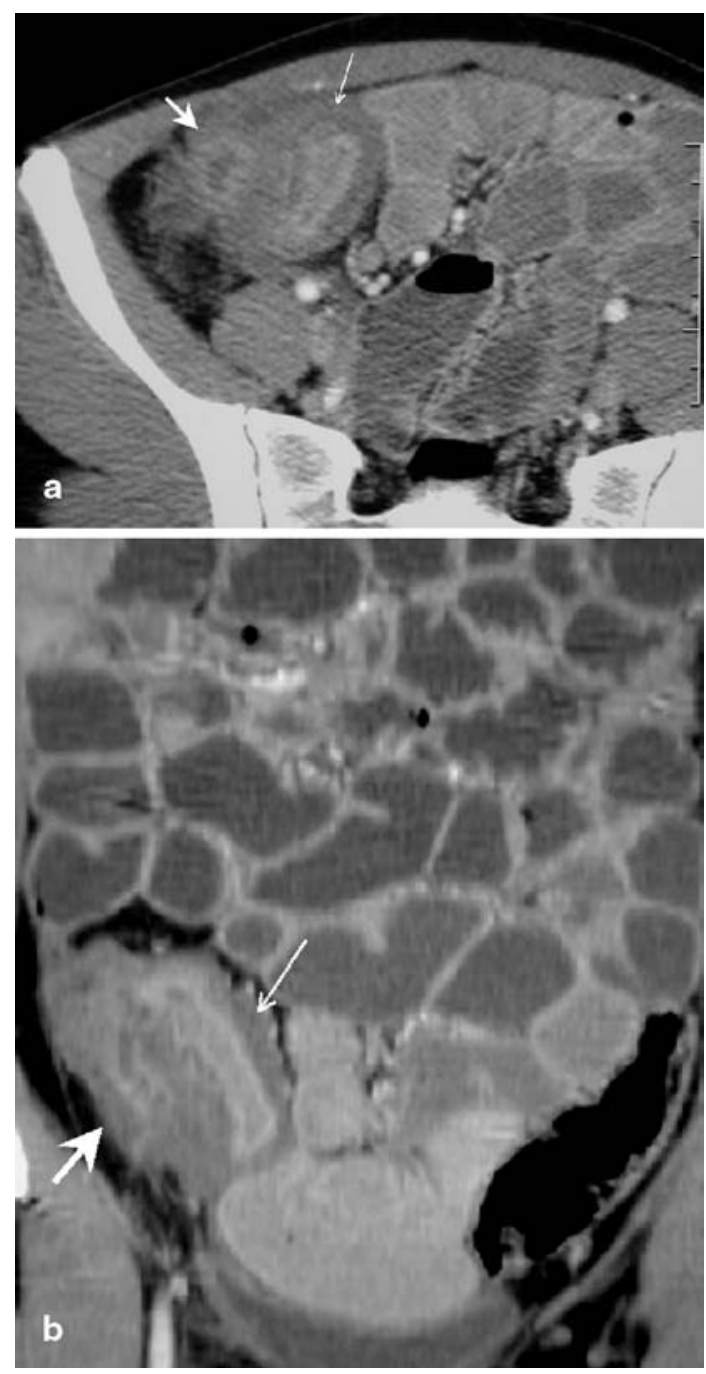

Fig. 6 a,b MDCTE in a 22-year-old woman with acute Crohn's disease: important parietal thickening of the distal ileum (thin arrow) and surrounding inflammation causes luminal stenosis complicated by an intraperitoneal abscess (thick arrow), both seen on a axial and $\mathbf{b}$ coronal views; however, coronal reconstructions better demonstrate the exact length of stenosis as well as the anatomical abscess extension

Interobserver agreement, sensitivity and specificity resulting from the independent and blinded evaluation by the three radiologists are displayed in Table 2. It deals with the results of the six most common pathological signs, because the incidence of the other six was too small to be informative. Axial and coronal slices both showed good interobserver agreement in all six radiological features with kappa ranging from 0.41 to 0.69. Kappa values for axial slices were superior to coronal reconstructions in four out of six pathological signs (BWT: 0.61 vs. 0.44 , BWE: 0.56 vs. 0.5 , ADP: 0.69 vs. 0.65 , IPF: 0.53 vs. 0.43 ). Coronal reformatted images only demonstrated better interobserver agreement for "adja-

cent mesenteric fat infiltration" and "bowel stenosis" (MFI: 0.66 vs. 0.57 , BST: 0.51 vs. 0.41 ). The average kappa on axial was slightly superior to coronal slices (0.56 vs. 0.52).

Sensitivity for each of the six signs was better on axial than coronal slices, ranging from $75.9 \%$ for BWE to $85.2 \%$ for ADP, so it was true for average sensitivity $(77.8 \%$ vs. $69.4 \%)$. The largest discrepancy was seen in IPF $(80.3 \%$ vs. $64.3 \%)$ followed by MFI (65.6\% vs. $77.5 \%)$.

Three out of the most common six pathological features demonstrated better specificity on axial slices (MFI: $97.9 \%$ vs. $95.1 \%$, ADP: $96 \%$ vs. $93.5 \%$ and IPF: $99.3 \%$ vs. $98.7 \%$ ), whereas specificity was superior on coronal reconstructions for the other three signs (BWT: $85 \%$ vs. $81.7 \%$, BST: $94.7 \%$ vs. $91.2 \%$ and BWE: $90.6 \%$ vs. $88.9 \%$ ). Average specificity was nearly equal on both views $(92.4 \%$ vs. $92.9 \%)$.

The Wilcoxon signed-rank test applied on the six most common pathological signs revealed significantly better sensitivity for axial slices than for coronal reconstructions $(P=0.0453)$, whereas there were no significant differences in specificity.

According to Student's $t$ test, the three observers interpreted axial slices with a significantly higher level of confidence $(P=0.0029)$ than the coronal reconstructions (mean \pm standard error: $1.18 \pm 0.44$ vs. $1.28 \pm 0.58$ ).

\section{Discussion}

The technique of multi-detector row CT was developed 6 years ago, and it has been improving constantly [19]. The volume coverage speed of multidetector row helical CT is twice to three times as fast as in single-detector row helical CT, with diagnostically comparable image quality [20]. This shorter acquisition time and better spatial resolution regarding the $z$ axis have improved the quality of multiplanar reconstructions, which is of particular advantage for the investigation of intestinal pathologies [20, 21]. Therefore, we observed an extension of indications, such as virtual colonoscopy [22], intestinal ischemia [23], including simultaneous CT angiography [21] and entero-CT, respectively, MDCTE [13-15, 24]. By excluding all technically suboptimal MDCTE, we ensured a good quality of imaging, especially complete small bowel opacification associated with good luminal distension in all patients.

We used dual-phase acquisition because parietal vascular malformations explaining subacute gastrointestinal bleeding are better seen in the arterial phase (at $30 \mathrm{~s}$ ) of MDCTE. The venous phase (at $60 \mathrm{~s}$ ) then allows detailed analysis of the intestinal wall, for example, inflammatory mucosal enhancement contrasting with adjacent submucosal edema $[15,24]$, provided that water or any other neutral enteral contrast medium is used, such as methyl- 
cellulosis in our study. Opacified mesenteric vessels can be analyzed exactly on the coronal plane, sometimes completed by $3 \mathrm{D}$ angiography, without being obscured by high-density bowel content resulting from the administration of positive enteral contrast agent [21, 25]. The detection of small luminal subtraction images such as polyps (Fig. 2) equally mostly enhancing with i.v. contrast medium [11] is also easier with the use of neutral enteral contrast material. According to Orjollet et al., monodetector CT enteroclyis is not the best modality for the evaluation of certain polyps as they occur, for example, in familial polyadenomatosis, because these small and low-enhancing adenomas are often mistaken for Kerckring folds on axial slices of $5 \mathrm{~mm}$ thickness [11]. MDCTE with thinner acquisition of axial slices and immediate coronal reconstructions now should allow us to overcome this problem. However, the exact role of multiplanar reconstructions in small bowel disease has not yet been evaluated on any large study population to our knowledge.

In a pictorial essay, Caoili et al. considered multiplanar reconstructions as a new perspective for the evaluation of small bowel obstruction: Using a helical single-detector $\mathrm{CT}$ and a collimation of $5 \mathrm{~mm}$ (pitch 1.5), axial images were reconstructed at 3-mm intervals in axial and coronal planes. According to the authors, coronal reformations increase diagnostic confidence and confirm the information revealed on axial slices, however, without proven efficacy and adding the own diagnostic value so far [26].

Raptopoulos et al. retrospectively evaluated the additional diagnostic value of multiplanar and especially coronal projections to axial slices [27] in 22 patients. They equally used a pitch of $1.5-$ and $5-\mathrm{mm}$ collimation with a slice increment of $3 \mathrm{~mm}$. Additional coronal reconstructions did not reveal further intestinal abnormalities, but significantly improved observers' confidence in image interpretation, particularly in their assessment of complicated Crohn's disease. In our study, radiologists' confidence remained significantly higher in their interpretation of axial slices than of coronal reconstructions alone, certainly due to the fact that radiologists are still more familiar with axial planes on CT. It remains the initial view in which images are displayed in the daily routine [28]. Certainly radiologists' confidence will even be reinforced by looking at both planes together [27], but this evaluation was beyond the aim of our study.

However, the higher diagnostic confidence obtained by Raptopoulos et al. also means a longer time of interpretation spent on one patient since its overall number of slices increases, including coronal reconstructions [27]. The use of work stations instead of film reading has meanwhile become mandatory in order to deal correctly with the large data sets radiologists have to face nowadays $[21,29]$. Our retrospective study showed a significantly shorter time of reading for coronal reconstructions than for axial slices, certainly because the former provided fewer images, about half of the axial view. Therefore, replacement of axial images by coronal reconstructions would be the ideal and time-appropriate method for image analysis for the future.

Prokesch et al. compared transverse images with curved coronal reformations for the staging and resectability of pancreatic carcinoma [28]. Average sensitivity and specificity between the two techniques concerning tumor detection and resectability were statistically equivalent, average interpretation time being much shorter for reformatted images due to their limited number compared to axial slices. These authors equally used a four-row multidetector CT and a dual phase i.v. injection pancreatic protocol with thin collimation of $4 \times 1.2 \mathrm{~mm}$ during the pancreatic phase, from which the reformatted images were derived. This acquisition technique allowed for more overlapping image reconstruction than in our study, but the authors had to cover the pancreatic region only, therefore using a far shorter $z$ axis than in our case. Secondly, Prokesch et al. centerd the curved planar reformations on specific anatomical structures only, such as the mesenteric vessel and pancreatic duct, etc., with the objective to complete and not to replace transverse images at the end, even if-for study purposes - both techniques were separately evaluated by three independent radiologists. Our coronal reconstructions, intended to be exhaustive, had to include a much larger volume of interest. They had to display the entire abdominal cavity from diaphragm to symphysis of $40-60 \mathrm{~cm}$ of length in order to include all the small bowel loops and the adjacent mesentery. Besides, creation of curved planar images required trained full-time radiographers under the guidance of experienced radiologists taking about $20 \mathrm{~min}$ for each case, whereas our coronal reformatted slices were semi-automatically reconstructed within seconds. Prokesch et al. point out the important clinical utility of these curved planar reformations, providing an excellent and time-efficient overview of pertinent findings especially for referring surgeons [28].

Our results demonstrate a good interobserver agreement, between 0.41 and 0.7 concerning the six most common pathological features on axial slices as well as on coronal reconstructions. However, sensitivity for all the six most common signs and interobserver agreement for four out of six features were inferior on coronal slices compared to the axial view.

At advanced stages of Crohn's disease, coronal reconstructions are considered to be especially useful [27] to define the anatomical extension of the inflammatory process, to detect fistulae extending in the axial plane and to characterize the exact length of inflammatory or fibrotic segmental stenoses of the small bowel. The latter is confirmed by the better interobserver agreement for bowel stenosis that we obtained for coronal slices compared to axial images and demonstrated in Fig. 5, 6. The pathological feature of adjacent mesenteric infiltration also showed better interobserver agreement on coronal recon- 
structions, but a significantly lesser sensibility than on axial slices. Figure 5 demonstrates how coronal views can underestimate the extension of transmural inflammation in Crohn's disease, such as vascular hyperemia and mesenteric edema. This observation agrees with the results of Raptopoulos et al., which showed inflammatory bowel involvement to be less extensive on coronal reconstructions than when assessed by axial slices alone [27]. The typical target or double halo appearance of inflammed small bowel loops might more easily be detected on the perpendicular view of axial images than on the coronal view where the bowel wall is mostly cut along its long axis. Therefore, our results indicates a better interobserver agreement and sensitivity for bowel wall thickening and pathological bowel wall enhancement on axial images than on coronal reconstructions.

In our study, the pathological sign lymphadenopathy showed altogether the best kappa and highest sensitivity of our six most common pathological features concerning the axial slices as well as the coronal reconstructions. The excellent spatial resolution with multidetector CT now allows the detection of lymph nodes of even $3 \mathrm{~mm}$ of smallest diameter in the abdominal cavity.

The largest statistical discrepancy between the two views concerns intraperitoneal fluid. In fact, Fig. 4 shows that small amounts of pelvic ascites were particularly difficult to detect on coronal reconstructions, despite the better display of the abdominal cavity in the coronal plane.

\section{Limitations of the study}

We chose the axial view in combination with the histopathological result, respectively, long-term follow-up of patients as the gold standard. We had to rely on clinical evolution in nine patients $(18.7 \%)$. Since non-invasive investigation of the small bowel is still difficult to perform, there are only a few other imaging modalities that could serve as a comparison, such as MRI enteroclysis (MRE). In a recent prospective study, MDCTE revealed diagnostic superiority over MRE because of better sensitivity and interobserver agreement in detecting small bowel lesions [15]. MRE allows primary coronal views as a multiplanar modality, but the time of image acquisition is still far longer and spatial resolution still remains inferior compared to MDCTE.

As proven and explained by means of our quality assurance test detailed above (Fig. 1a,b,c), our coronal reconstructions were of lower spatial resolution than axial slices. This difference in spatial resolution means that the coronal views obtained in our study should not be allowed to separate (in the worst case) two structures that are less than $4 \mathrm{~mm}$ apart (Nyquist frequency of $0.25 \mathrm{~mm}^{-1}$ ), whereas in the transverse view it should be possible to discriminate structures that are $1.4 \mathrm{~mm}$ apart and above (i.e., Nyquist frequency of $0.7 \mathrm{~mm}^{-1}$ ). It should be pointed out, however, that a lesion smaller than $2 \mathrm{~mm}$, but highly contrasted, could be detected in the coronal views obtained in our conditions. However, its perceived contrast would be reduced due to pixel partial volume effects.

However, since the diameter of our smallest pathological features was never below $2 \mathrm{~mm}$, we accepted this drawback for practical reasons as being within the limitations of a retrospective study, since we could not change the applied acquisition parameters, especially the pitch factor, any more.

Furthermore, compared with other possibilities of image data reconstruction, multiplanar reformations still remain a superior diagnostic tool, especially for small low contrast lesions (diameter of $3 \mathrm{~mm}$ and less), than volume-rendering techniques, which are particularly suited for larger objects and data with high noise content [30].

The only possibility to improve the spatial resolution of the coronal views in our retrospective study was therefore the reduction of the slice increment to a value close to half of the acquisition slice thickness (i.e., lower than $1.25 \mathrm{~mm}$ ). Figure $1 \mathrm{c}$, however, shows that coronal reformatting with a slice increment of $1.2 \mathrm{~mm}$ does not significantly improve spatial frequency.

The narrower the axial collimation and the smaller the pitch of MDCTE chosen, the better the spatial resolution and also the coronal views will be due to sampling improvements [21]. The thinnest possible slice thickness on four-array-detector CT is $1.25 \mathrm{~mm}$. This collimation, associated with a pitch value of 0.75 and a slice increment of $0.6 \mathrm{~mm}$, would have allowed the improvement of the spatial resolution in the coronal plane. From sampling properties, the limiting spatial frequency in the coronal plane would have been $0.8 \mathrm{~mm}^{-1}$ (i.e., $(1 / 0.6) \times 0.5 \mathrm{~mm}^{-1}$ ), but such a long image acquisition would have significantly increased the risk of bowel movement and respiration artifacts.

This inconvenience has now been overcome with the newer generation of 16-array-detector CT, since thinnest slices of $0.63 \mathrm{~mm}$ are possible. Using parameters of $16 \times 0.63 \mathrm{~mm}$ at a pitch of 1.35 , therefore covering a volume of $13.6 \mathrm{~mm}$ per second, image acquisition then becomes really isotropic, and multiplanar reformatting (MPR) with a slice increment smaller than half of the reconstructed slice thickness can easily be obtained.

\section{Conclusion}

In summary, our study confirms the present superior diagnostic value of axial slices over coronal reconstructions because of better sensibility in our six most common pathological features and higher interobserver agreement in most of them. Diagnostic confidence is equally superior for axial images, each plane being analyzed separately, certainly even increasing when looking at both views together. 


\section{References}

1. Maglinte DD, Kelvin F, O'Connor K (1996) Current status of small bowel radiography. Abdom Imaging 2:247-257

2. Klöppel R, Thiele J, Bosse J (1992)

The sellink CT method. RöFo 156:291-292

3. Bender GN, Maglinte DD, Klöppel R, Timmons JH (1999) CT-enteroclysis: a superflous diagnostic procedure or valuable when investigating smallbowel disease? Am J Roentgenol 172:373-378

4. Rieber A, Wruk D, Nüssle K, Aschoff AJ, Reinshagen M, Adler G et al (1998) MRT des Abdomens in Kombination mit der Enteroklyse bei Morbus Crohn unter Verwendung von oralem und intravenösem Gd-DTPA. Radiologe 38:23-28

5. Maglinte DD, Siegelman ES, Kelvin FM (2000) MR Enteroclysis: the future of small-bowel imaging? Radiology 215:639-641

6. Maglinte DD, Bender GN, Heitkamp DE, Lappas JC, Kelvin FM (2003) Multidetector-row helical CT enteroclysis. Radiol Clin North Am 41(2):249-262

7. Bender GN, Timmons JH, Williard WC, Carter J (1996) Computed tomographic enteroclysis-one methodology. Invest Radiol 31(1):43-39

8. Thiele J, Klöppel R, Schulz HG (1993 CT-Sellink-eine neue Methode der Darmwandbeurteilung. RöFo 159(3):213-217

9. Schober E, Turetschek K, Schima W, Mostbeck GH (1997) Methylcellulose spiral CT in the preoperative assessment of Crohn's disease: radiologic and pathologic correlation (abstract) Radiology 205(P):717
10. Walsh DW, Bender GN, Timmons JH (1998) Comparison of computed tomography-enteroclysis and traditional computed tomography in the setting of suspected partial small bowel obstruction. Emerg Radiol 5:29-37

11. Orjollet-Lecoanet C, Menard Y, Martins A, Crombé-Ternamian A, Cotton F, Valette PJ (2000) L'Enteroscanner: Une nouvelle méthode d'exploration du grêle. J Radiol 81:618-627

12. Gaffke G, Stroszczynski C, Schlecht I, Reiche K, Wickede M von, Ludwig WD, Felix R (2001) Diagnostik von Dünndarmtumoren mit Hilfe der CT-Sellink Methode (abstract). RöFo 173:S37

13. Rust GF, Holzknecht N, Olbrich D,Schöpf U, Brüning R, Reiser M (1999) Mehrschicht-Computertomographie des Dünndarms - vorläufige Ergebnisse. Radiologe 39:965-970

14. Rust GF, Spiekermann A, Daum F, Schoepf UJ, Holzknecht N, Matz C, Staebler A, Reiser MF (2001) New developments in imaging the small bowel with multislice computed tomography and negtive contrast medium. In: Reiser MF (ed) Multislice CT. Springer, Berlin Heidelberg New York, pp 49-60

15. Schmidt S, Lepori D, Meuwly JY, Duvoisin B, Meuli R, Michetti P et al (2002) Prospective comparison of MRenteroclysis (MRE) with multidetectorspiral-CT-enteroclysis (MSCTE). Eur Radiol 13:1303-1311

16. Silverman PM, Kalender WA, Hazle JD (2001) Common terminology for single and multislice helical CT. Am J Roentgenol 165(5):1135-1136

17. Kalender WA, Seissler W, Klotz E, Vock P (1990) Spiral volumetric CT with single-breath-hold technique, continuous transport, and continuous scanner rotation. Radiology 176(1):181-183

18. Fleiss JL (1985) Statistical methodes for rates and proportions. Wiley, New York

19. Kalender WA (2000) Computertomographie. Publicis MCD Verlag, München

20. $\mathrm{Hu} \mathrm{H}, \mathrm{He} \mathrm{HD}$, Foley WE, Fox SH (2000) Four multidetector row helical CT: image quality and volume coverage speed. Radiology 215:55-62
21. Ros PR, Ji H (2002) Multisection (multidetector) CT: applications in the abdomen. Radiographics 22:697-700

22. Luboldt W, Fletcher JG, Vogl TJ (2002) Colonography: current status, research directions and challenges. Update 2002 (review). Eur Radiol 12:502-525

23. Horton KM, Fishman EK (2001) Multi-detector row CT of mesenteric ischemia: can it be done? RadioGraphics 21:1463-1473

24. Bruel JM, Gallix B (2003) Scanner multidétecteur face à l'IRM dans la pathologie du tube digestif. J Radiol 84:499-513

25. Horton KM, Fishman EK (2003) The current status of multidetector row CT and three-dimensional imaging of the small bowel. Radiol Clin North Am 41(2):199-212

26. Caoili EM, Paulson EK (2000) CT of small-bowel obstruction: another perspective using multiplanar reformations. Am J Roentgenol 174:993-998

27. Raptopoulos V, Schwartz RK McNicholas MMJ, Movson J, Pearlma J, Joffe N (1997) Multiplanar helical CT enterography in patients with Crohn's disease. Am J Roentgenol 169:1545-1550

28. Prokesch RW, Chow LC, Beaulieu CF, Nino MM, Mindelzun RE, Huang J, Jeffrey RB (2002) Local staging of pancreatic carcinoma with multidetector row CT: use of curved planar reformations-initial experience. Radiology 225:759-765

29. Foley WD (2002) Multidetector CT: abdominal visceral imaging. Radiographics 22:701-771

30. Shin HO, Falck CV, Galanski M (2004) Low-contrast detectability in volume rendering: a phantom study on multidetector-row spiral CT data. Eur Radiol 14(2):341-349 